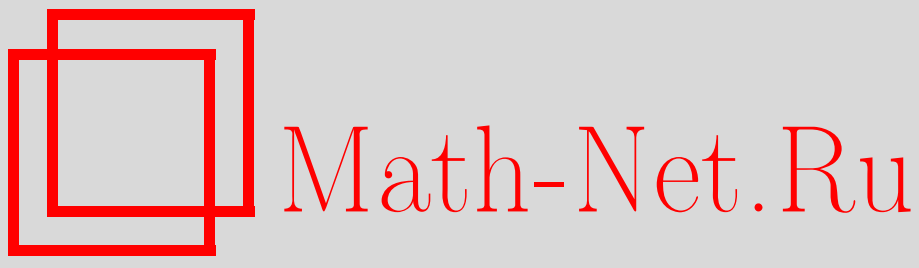

А. М. Шур, Ю. В. Гамзова, Частичные слова и свойство взаимодействия периодов, Изв. РАН. Сер. матем., 2004, том 68, выпуск 2, 191-214

DOI: https://doi.org/10.4213/im480

Использование Общероссийского математического портала Math-Net.Ru подразумевает, что вы прочитали и согласны с пользовательским соглашением

http://www. mathnet.ru/rus/agreement

Параметры загрузки:

IP : 3.85 .73 .92

26 апреля 2023 г., 16:41:52 
УДК $512.532 .2+519.11$

А. М. Шур, Ю.В. Гамзова

\section{Частичные слова и свойство взаимодействия периодов}

Теорема Файна-Вильфа выражает фундаментальное свойство периодических слов - свойство взаимодействия периодов. Произвольное слово с периодами $p$ и $q$ имеет произвольный период, равный НОД $(p, q)$, если длина слова больше критической величины, называемой длиной взаимодействия. В настоящей работе рассмотрено аналогичное свойство для произвольных периодических частичных слов и получена точная линейная оценка длины взаимодействия.

Библиография: 7 наименований.

\section{$\S 1$. Введение}

Частичное слово представляет собой комбинаторньй объект, получаемый естественным обобщением понятия обычного конечного слова. Слово, т.е. конечную последовательность элементов фиксированного множества (алфавита), можно рассматривать как функцию из множества $\{1, \ldots, n\}$ в алфавит. Тогда частичное слово длины $n$ над алфавитом $A$ может быть определено (см. [1]) как частичная функция

$$
W:\{1, \ldots, n\} \rightarrow A .
$$

Интерес к комбинаторным свойствам частичных слов возник совсем недавно и продиктован потребностями компьютерных наук и молекулярной биологии. Систематическое исследование частичных слов только начинается.

Задачи, в которых возникают частичные слова, можно разделить на два типа. В задачах первого типа некоторая часть информации нам не важна (вне зависимости от того, доступна она или нет). Примером может служить задача поиска в тексте по шаблону, когда шаблон содержит символ (или символы) со значением "что угодно". В задачах второго типа некоторая часть информации для нас важна, но по каким-либо причинам недоступна. По имеющейся части информации и некоторым дополнительным условиям необходимо полностью восстановить информацию. Примером является задача восстановления фрагментов файлов, поврежденных при передаче или в результате порчи носителя. Очень интересными представляются также задачи молекулярной биологии (см. [7]), в частности задача реконструкции нуклеотидных цепочек ДНК по частично расшифрованныгм фрагментам.

В последние годы начато изучение периодических частичных слов (см. [1], [4], [5]), но пока только в связи с одним известным комбинаторным свойством - свойством взаимодействия периодов, выражаемым для обычных слов хорошо известной теоремой Файна-Вильфа (см. [2], а также [3]). Анализу этого свойства посвящена 
и данная работа. Однако представляет интерес обобщение на случай частичньх слов и других свойств периодических слов, например, теоремы о критическом разбиении [3].

Отметим еще два направления в комбинаторике слов, рамки которых разумно расширить, включив частичные слова в объекты исследования. Первое из них связано с теорией избегаемости. Можно заметить, что избегаемые слова ("шаблоны") представляют собой частичные слова в несколько завуалированном виде. Соответственно, изучение свойств частичных слов в этом направлении позволит переосмыслить и расширить сушествующую теорию. Второе направление связано с изучением структуры и свойств множества подслов ("делителей") данного слова и содержит некоторые задачи молекулярной биологии (см., например, [6]). В работе [1] упоминается проблема "наложения" частичных слов, лежащая в рамках этого направления.

Приведем необходимые определения для обычных слов. Длина слова $W$ есть количество символов в этом слове (обозначается через $|W|$ ). Порядковый номер символа в слове называется его позииией. Натуральное число $p$, не превосходящее $|W|$, называется периодом слова $W$, если

$$
W(i)=W(i+p), \quad i=1, \ldots,|W|-p .
$$

В частности, любое слово имеет тривиальный период, равный его длине.

Далее будем рассматривать слова, имеющие по крайней мере два различных периода. Для периодических слов одним из основных свойств является свойство взаимодействия периодов: достаточно длинное слово с периодами $p$ и $q$ имеет также "производный" период НОД $(p, q)$. Точная формулировка приводится в теореме Файна-Вильфа [2], которую мы сформулируем здесь в удобных для нас обозначениях.

ТеОРема 1.1. Пусть р и - натуральные числа. Тогда каждое слово длины не менее чем $p+q-\mathrm{HОД}(p, q)$ с периодами $p$ и q имеет период НОД $(p, q)$. Указанная оченка является точной.

Через $D(W)$ обозначим область определения частичной функции $W(i)$. Каждому частичному слову $W$ над алфавитом $A$ можно поставить в соответствие обычное слово $W_{\diamond}$ над расширенным алфавитом $A_{\diamond}=A \cup\{\diamond\}$ следуюшим образом:

$$
W_{\diamond}(i)= \begin{cases}W(i), & i \in D(W), \\ \diamond, & i \notin D(W) .\end{cases}
$$

Отображение $W \rightarrow W_{\diamond}$ является биекцией. Далее под частичным словом будем понимать именно конечную последовательность символов над алфавитом $A \cup\{\diamond\}$, используя, в частности, те же определения длины и позищии, что и для обычных слов.

Значение символа $\diamond$ таково: "на этой позиции должен находиться алфавитный символ, неизвестно какой" . (При рассмотрении некоторых задач, например задачи поиска в тексте, "неизвестно" заменяется на "безразлично", но наша задача к таким не относится, как станет ясно из определений.) В статье [1] символ $\diamond$ назывался дырой. Мы используем термин джскер, который кажется нам более уместным. Отличные от джокера символы алфавита будем, как обычно, называть буквами. 
В работе [1] рассмотрены две естественные возможности обобщения свойства периодичности на частичные слова. Приведем соответствующие определения.

Частичное слово $W$ имеет период $p$, если

$$
i \equiv j(\bmod p) \Longrightarrow W(i)=W(j), \quad i, j \in D(W) .
$$

Например, слово $W=a b \diamond a \diamond c a b c$ имеет период 3 .

Частичное слово $W$ имеет локальный период $p$, если

$$
W(i)=W(i+p), \quad i, i+p \in D(W) .
$$

Для обычных слов наличие локального периода $p$ всегда влечет наличие периода $p$, но это свойство не выполняется для частичных слов. Например, частичное слово $W=a b c \diamond b c d$ имеет локальный период 3 , но не имеет периода 3 . Заметим, что частичное слово имеет период $p$ тогда и только тогда, когда в нем все джокеры можно заменить буквами так, что полученное обычное слово будет иметь период $p$.

В [1] был доказан аналог теоремы 1.1 для локально периодических частичных слов с одним джокером.

ТеОрема 1.2 [1]. Пусть р и q-натуральные числа. Тогда любое частичное слово длины не менее чем $p+q$ с одним джокером и локальными периодами $p$ u q имеет период НОД $(p, q)$. Указанная оченка является точной.

Однако если частичное слово содержит более одного джокера, то свойство взаимодействия локальных периодов может вообще не выполняться. Например, частичное слово $U$ с периодами $p$ и $q$ может иметь два джокера в позициях $q+1$ и $p+1$ и различные буквы в позициях 1 и НОД $(p, q)+1$. Следовательно, для локально периодических слов с более чем одним джокером можно доказывать лишь "условные" теоремы. Соответствуюшие оценки для случаев двух и трех джокеров приведены в [5].

Таким образом, чтобы получить результаты, аналогичные теореме 1.1, для произвольных частичных слов следует использовать условия, связанные с периодами, а не с локальными периодами.

Итак, в связи с частичными словами и свойством взаимодействия периодов интерес представляют следующие вопросы.

1. Верно ли, что частичное слово достаточно большой длины, имеющее периоды $p$ и $q$ и фиксированное число джокеров $k$, обязательно имеет период НОД $(p, q)$ ?

2. Если ответ на первьй вопрос - утвердительный, то какой длины должно быть частичное слово, имеющее периоды $p, q$ и содержащее $k$ джокеров, чтобы оно также гарантированно имело период НОД $(p, q)$ ? (Тривиальный случай длины $k+1$ исключим из рассмотрения.)

Наименьшую такую длину будем называть длиной взаимодействия периодов $p$ u q при наличии $k$ джокеров и обозначать $L(k, p, q)$. Длину взаимодействия естественно рассматривать как функцию от $k$ с параметрами $p$ и $q$.

В работе [4] был получен утвердительный ответ на первый вопрос и частичный ответ на второй. Полньй ответ на второй вопрос является целью настоящей работы.

7 Серия математическая, №2 


\section{§2. Основной результат работы}

Как отмечалось в некоторых работах (см. [1], [3], [4]), утверждения о взаимодействии произвольных периодов $p$ и $q$ тривиально следуют из аналогичных утверждений для взаимно простых периодов ввиду следующего наблюдения.

НАБЛЮДЕНИЕ. В случае, когда НОД $(p, q)=d>1$, можно заменить (частичное) слово $U$ набором, состояшим из $d$ (частичных) слов $U_{1}, \ldots, U_{d}$, где $U_{i}=$ $U(i) U(d+i) U(2 d+i) \ldots$. Каждое из этих слов будет иметь взаимно простые периоды $p / d, q / d$. При этом слово $U$ будет иметь период $d$ тогда и только тогда, когда все $U_{i}$ имеют период 1.

Таким образом, в настоящей работе мы исследуем длину взаимодействия взаимно простых периодов. Перечислим известные ранее оценки длины взаимодействия в предположении, что $p>q$ (знак \lfloor\rfloor здесь и далее обозначает нижнее целое):

1) $L(0, p, q)=p+q-1 \quad($ см. $[2])$;

2) $L(1, p, q)=p+q \quad$ (см. $[1])$;

3) $L(2, p, q)=p+2 q-1 \quad$ (cм. [4]);

4) $L(k, p, 2)=(2 p+1)\lfloor k / p\rfloor+k \bmod p \quad$ (cм. [4]);

5) $L(k, p, q) \leqslant q k+p+q-1 \quad$ (cм. [4]).

Кроме того, в работе [4] было доказано, что оценка (2.5) является точной при $k \neq 0$ только в случае, когда $q=2$ и $p$ делит $k$; во всех остальных случаях эта оценка может быть улучшена. Из этого доказательства немедленно следовало и не отмеченное явно в [4] равенство (2.4).

Таким образом, длина взаимодействия известна точно в нескольких простейших случаях и оценена сверху в общем случае линейной по $k$ функцией с коэффициентом $q$. Эти результаты, на наш взгляд, исчерпали возможности использовавшейся комбинаторной техники, основанной на графах бинарных отношений. В настоящей работе предложена иная техника, позволяющая найти оптимальную линейную оценку в общем случае. С учетом (2.4) можно предполагать, что $q \geqslant 3$. Основным результатом работы является следующая теорема.

TЕОРема 2.1. Пусть р и $q$-взаимно простые периоды слова с $k$ джскера$\mathcal{M} u, p>q \geqslant 3, \quad k \geqslant\lfloor 2 p / 3\rfloor-1$ npu $q=3 u k \geqslant\lfloor 3 p / q\rfloor+3$ npu $q \geqslant 4, \quad L(k, p, q)-$ длина взаимодействия периодов р и п при наличии $k$ джокеров. Тогда:

а) $\frac{p q}{p+q-2}(k+1) \leqslant L(k, p, q)<\frac{p q}{p+q-2} k+4(q-1)$;

b) функиия $\Delta(k, p, q)=L(k, p, q)-\frac{p q}{p+q-2} k$ является периодической по $k$ с периодом $p+q-2$;

c) для любого $\varepsilon>0$ и любого q найдется $p$ такое, что

$$
\max \Delta(k, p, q)>4(q-1)-\varepsilon ;
$$

d) วля любьх $p$ u

$$
\min \Delta(k, p, q)=\frac{p q}{p+q-2}
$$

е) для любьх $p u q$

$$
\max \Delta(k, p, q) \geqslant 2 q .
$$


ПРимеР 2.1. График зависимости длины взаимодействия от числа джокеров при $p=13, q=3$ вместе с графиками верхней и нижней оценок из теоремы 2.1 приведены на рис. 1. Отметим, что согласно теореме 2.1 эти оценки верны при $k \geqslant 7$.

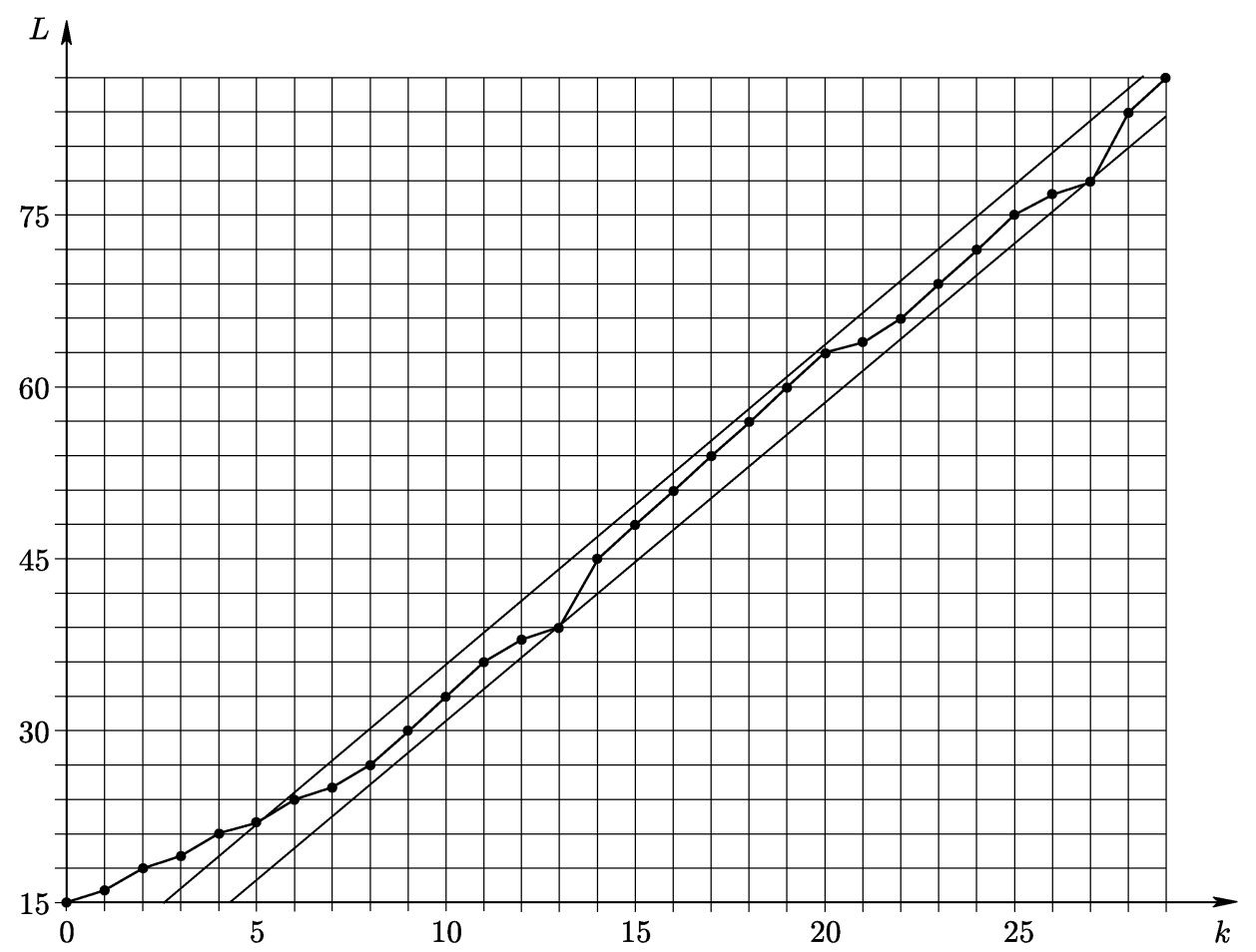

Рис. 1. График зависимости длины взаимодействия от числа джокеров

\section{§3. Обратная задача. Бланки и расстановки джокеров}

Далее под словом мы всюду понимаем частичное слово, а $p$ и $q$ считаем взаимно простыми числами такими, что $p>q \geqslant 3$. Для доказательства основной теоремы рассмотрим обратную задачу: заданы длина слова $L$ и его периоды $p, q$, необходимо найти минимальное количество джокеров, при котором слово может не иметь периода 1.

Заметим, что для каждого периода $p$ произвольного слова $U$ и для любого $i$, $1 \leqslant i \leqslant p$, все буквы, находящиеся в позициях $i, i+p, i+2 p, \ldots$, равны независимо от расположения джокеров в этих позициях. Указанное множество позиций мы будем называть $P$-классом; оно содержится в $i$-м классе вычетов по модулю $p$.

Рассмотрим случай двух различных периодов $p, q$ слова $U$, и, соответственно, будем рассматривать $P$ - и $Q$-классы. Заметим, что если две позиции из разных $Q$-классов, занятые буквами, принадлежат одному $P$-классу (т.е. "связаны” при 
помощи периода $p$ ), то в этих $Q$-классах содержится одна и та же буква. Если же любая пара позищий из данных $Q$-классов, принадлежащая одному $P$-классу, содержит хотя бы один джокер, то эти $Q$-классы могут содержать различные буквы.

Таким образом, чтобы построить слово $U$ длины $L$ с периодами $p$ и $q$, содержащее $m$ различных букв $(m \leqslant q)$, нужно разбить $Q$-классы (их количество равно $q$ ) на $m$ групп и разместить джокеры так, чтобы период $p$ связьвал между собой только такие позиции, не занятые джокерами, которые принадлежат $Q$-классам из одной группы.

Чтобы решить поставленную обратную задачу, необходимо найти наиболее экономный способ размещения джокеров для случая $m=2$. Процедура построения слова $U$ в этом случае будет выглядеть следующим образом. Разделим $Q$-классы на 2 группы: $T$ и $S$, содержащие соответственно $t$ и $s$ классов $(0<t \leqslant s, t+s=q)$. Для каждого $P$-класса выберем одну из груп $T$ или $S$ и разместим джокеры во всех позициях этого $P$-класса, принадлежащих к $Q$-классам из выбранной группы. Если мы хотя бы по разу выбрали каждую из групп, то в $Q$-классах как из $T$, так и из $S$ останутся позиции, не занятые джокерами. В них мы разместим буквы (одну для $T$, другую для $S$ ) и тем самым получим слово $U$, не имеюшее периода 1 . Несложно заметить, что число джокеров в $U$, которое мы стремимся минимизировать, зависит от $t$ и способа выбора групп для $Q$-классов.

Опуская детали, рассмотренную вьше процедуру можно представить следуюшим образом: имеется некоторый "шаблон” слова $U$, в котором мы по определенным правилам размещаем алфавитные символы (вначале - джокеры, а затем буквы). Интуитивное понятие "шаблона" мы сейчас оформим в виде специальной числовой таблицы (бланка), играющей ключевую роль в дальнейших рассмотрениях.

Запишем позиции слова $U$ в таблицу, как показано на рис. $2, a$.

Занумеруем $Q$-классы числами от 0 до $q-1$ (так, что $i$-й класс содержит позиции с номерами вида $i+n q)$ и заменим в приведенной таблице каждую позищию на номер содержащего ее $Q$-класса. Полученную таблицу будем называть бланком. Она имеет вид, изображенньй на рис. 2,6 (знак $\oplus$ обозначает сложение по модулю $q)$.

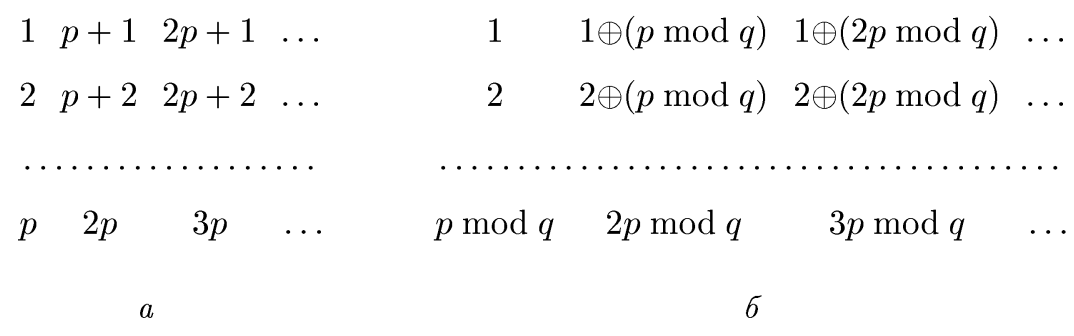

Рис. 2. Построение бланка

Для построения слова $U$ мы заменяем некоторые номера в строках (строки соответствуют $P$-классам) джокерами так, чтобы в любой строке оставались номера классов только из группы $S$ или только из группы $T$. 
ПРИмеР 3.1. Рассмотрим слово длины 15 , имеющее периоды 5 и 7 . Минимальное количество джокеров, при котором такое слово может не быть унарным, равно 2 (это следует из оценки (2.3)). Бланк для этого слова выглядит так, как показано на рис. $3, a$.

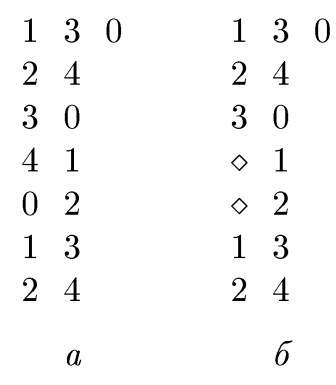

Рис. 3. Пример бланка и расстановки джокеров в нем

Легко убедиться, что наименьшее количество джокеров достигается при $T=$ $\{2,4\}$ и $S=\{0,1,3\}$. Тогда необходимо поставить по одному джокеру в 4 -ю и 5 -ю строки, например в позиции 4 и 5 (рис. 3,6 ). Теперь в каждой строке находятся номера $Q$-классов, принадлежаших одной группе. Заменив номера из $S$ на $a$, номера из $T$ на $b$ и записав буквы в обычном порядке, получим бинарное слово $a b a \diamond \diamond a b a b a a b a b a$.

ЗАмЕчАниЕ 3.1. В связи с рассматриваемой задачей нас интересует только стадия размещения джокеров в бланке (и последующий подсчет джокеров). Стадию размешения букв мы будем опускать.

ЗАмечаниЕ 3.2. Договоримся отождествлять номера $Q$-классов и сами $Q$-классы. В частности, будет удобнее рассматривать элементы множеств $S$ и $T$ как номера.

ЗАмечание 3.3. Параметры $L, p, q(L>p>q>1$, НОД $(p, q)=1)$ однозначно задают бланк. Поэтому бланк будем обозначать тройкой $(L, p, q)$.

Расстановкой джокеров в слове (бланке), или просто расстановкой, будем называть множество позиций в слове или в бланке, на которых стоят джокеры.

Пусть заданы периоды $p, q$ и длина $L$. Расстановку джокеров в бланке $(L, p, q)$ будем назьвать допустимой, если слово длины $L$ с периодами $p, q$ и указанной расстановкой джокеров может не иметь периода 1 (т. е. содержать две различные буквы). Допустимую расстановку с минимальным количеством джокеров будем называть оптимальной, а число джокеров в ней обозначать через $k^{*}$. Рассматриваемая нами обратная задача состоит, таким образом, в вычислении $k^{*}$.

Для данного $t$ (напомним, что $t=|T|$ ) рассмотрим все допустимые расстановки джокеров и выберем расстановку с минимальным количеством джокеров. Эту расстановку будем называть оптимальной при $t$, а количество джокеров в ней обозначать через $k^{*}(t)$.

Будем назьвать спещиальной расстановку следующего вида: $|T|=1$, во всех строках, кроме одной, джокерами заменяются все вхождения элемента из $T$ и только они, а в оставшейся строке - все вхождения элементов из $S$ и только они. Специальная расстановка, очевидно, является допустимой. 
Лемма 3.1. При $q \geqslant 3$ и $L \geqslant 2 p-2$ существует специальная расстановка в бланке $(L, p, q)$, оптимальная при $t=1$.

ДокАЗАТЕЛЬСТво. В бланке $(L, p, q)$ не более двух одноэлементных строк. Если такие строки существуют, поместим в множество $T Q$-класс, в них не встречаюшийся (такой найдется, поскольку $Q$-классов не менее трех); если таких строк нет, поместим в $T$ любой $Q$-класс. Теперь в каждой строке элементов из $T$ содержится не больше, чем элементов из $S$. Для фиксированного одноэлементного $T$ рассмотрим допустимую расстановку, в которой джокеры заменяют элементы $S$ более чем в одной строке. Заменив в одной из строк джокерами элементы $T$ вместо элементов $S$, вновь получим допустимую расстановку; количество джокеров при этом может лишь уменьшиться. Таким образом, при каждом фиксированном одноэлементном $T$ некоторая специальная расстановка содержит минимальное количество джокеров. Следовательно, найдется специальная расстановка, которая является оптимальной при $t=1$.

СЛЕДСТВИЕ 3.1. Для любого бланка $(L, p, 3)$ такого, что $L \geqslant 2 p-2, c y$ ществует оптимальная специальная расстановка.

ДоказАТЕЛЬСТво. Условие $q=3$ необходимо влечет $t=1$. Таким образом, специальная расстановка, оптимальная при $t=1$ (такая сушествует по лемме 3.1 ), является оптимальной.

Следуюшее предложение, позволяюшее решить обратную задачу, является ключевым в настоящей работе. Его доказательство связано с рядом технических трудностей, для преодоления которых использовались и нетрадиционные для комбинаторики методы: так, лемма 4.3 доказывается с использованием определенных интегралов.

ПреДЛОЖЕНИЕ 3.1. Для любого бланка $(L, p, q)$ такого, что $q \geqslant 4, L \geqslant$ $3 p+q$, существует оптимальная специальная расстановка.

ЗАмечаниЕ 3.4 . В примере 3.1 выполняется $k^{*}(1)=3$, и, таким образом, никакая специальная расстановка не оптимальна (но длина бланка в этом примере не удовлетворяет приводимым в предложении 3.1 ограничениям).

\section{§4. Решение обратной задачи}

Этот параграф̆ посвящен доказательству предложения 3.1, а также предложения 4.1, позволяюшего получить решение обратной задачи на основе подсчета джокеров в оптимальной специальной расстановке.

Для доказательства предложения 3.1 разделим бланк на части так, как показано на рис. 4.

Часть бланка, соответствующую префиксу слова длины $p q\left\lfloor\frac{L}{p q}\right\rfloor$, будем называть телом, а оставшуюся часть - хвостом. Тело состоит из $\left\lfloor\frac{L}{p q}\right\rfloor$ блоков - прямоугольников высоты $p$ и ширины $q-$ это части 1.

В хвосте выделим части $\mathbf{2}$ - это прямоугольники высоты $q$, ширина которых равна $\left\lfloor\frac{L \bmod p q}{p}\right\rfloor$ или $\left\lceil\frac{L \bmod p q}{p}\right\rceil$. Таких частей $\left\lfloor\frac{p}{q}\right\rfloor$ штук. Кроме того, в бланке 


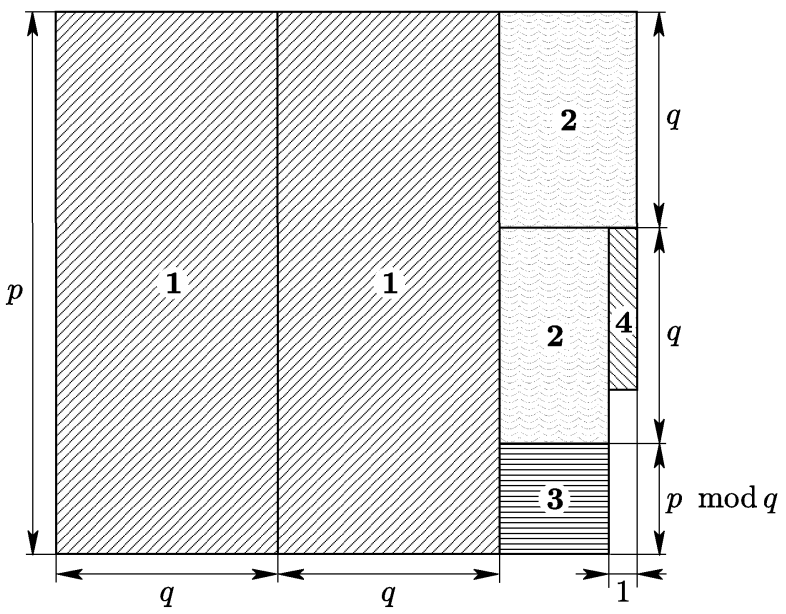

Рис. 4. Бланк

присутствуют одна часть 3 и одна часть 4 . Часть $\mathbf{3}$ - прямоугольник ширины $\left\lfloor\frac{L \bmod p q}{p}\right\rfloor$ и высоты $p \bmod q$. Часть 4 - прямоугольник ширины 1 и высоты $L \bmod q$, соответствующий суффиксу слова длины $L \bmod q$.

Определим расстановку для части $\mathbf{i}, \mathbf{i}=\mathbf{1}, \ldots, \mathbf{4}$, как пересечение части $\mathbf{i}$ и расстановки в данном бланке. Определения допустимой и оптимальной (при $t$ ) расстановок для части $\mathbf{i}$ аналогичны соответствуюшим определениям для расстановок. Количество джокеров в оптимальной при $t$ для части $\mathbf{i}$ расстановке будем обозначать через $k_{i}^{*}(t)$. Очевидно, что сумма значений $k_{i}^{*}(t)$ по всем частям бланка не превосходит $k^{*}(t)$.

Для доказательства предложения 3.1 рассмотрим отдельно количество джокеров в расстановках, оптимальных для каждой части. Лемма 4.2 посвящена исследованию части 1, леммы 4.2-4.4- части 2, лемма 4.5 - части 3. Рассмотрение расстановок для части 4 не представляет интереса: любая строка в этой части содержит только один элемент, а значит, оптимальная расстановка при любом $t$ вообще не содержит джокеров.

ЛЕмма 4.1. Для любого $2 \leqslant t \leqslant\lfloor q / 2\rfloor$ справедливо равенство

$$
k_{1}^{*}(t)-k_{1}^{*}(1)=(p-2)(t-1) .
$$

ДокаЗАТЕльство. Рассмотрим часть 1. Каждая строка блока является перестановкой множества $\{1, \ldots, q\}$. В самом деле, последовательность элементов первой строки имеет вид

$$
\pi=(1,1 \oplus(p \bmod q), 1 \oplus(2 p \bmod q), \ldots, 1 \oplus((q-1) p \bmod q)) ;
$$

все элементы $\pi$ различны, так как НОД $(p, q)=1$, а значит, $\pi$ является перестановкой множества $\{1, \ldots, q\}$. Остальные же строки являются циклическими перестановками $\pi$. Итак, в каждой строке блока номер каждого $Q$-класса встречается только один раз. Таким образом, каждая строка содержит $t$ элементов из $T$ и $s$ элементов из $S$. 
Хотя бы в одной строке мы должны разместить $t$ джокеров, хотя бы в одной $-s$ джокеров, и в каждой из оставшихся - либо $t$, либо $s$ джокеров. Поскольку $t \leqslant s$, то оптимальная расстановка для части 1 следующая: по $t$ джокеров в $p-1$ строке и $s=q-t$ джокеров в оставшейся строке. Тогда общее количество джокеров в части 1 при оптимальной для нее расстановке равно

$$
k_{1}^{*}(t)=(p-1) t+(q-t) .
$$

При $t \geqslant 2$ в части 1 необходимо поставить на $k_{1}^{*}(t)-k_{1}^{*}(1)=(p-2)(t-1)$ джокеров больше, чем при $t=1$.

Перейдем к рассмотрению части 2.

Отличительная особенность частей, принадлежащих хвосту бланка, состоит в том, что они пересекают не все строки бланка (т. е. не все $P$-классы). Это значит, что в расстановке, оптимальной для такой части, джокеры могут во всех строках заменять только элементы из $T$ : строки, в которых джокерами заменяются элементы из $S$, обязательно присутствуют в бланке, но могут не пересекать данную часть. Наличие таких строк будет учтено ниже, в доказательстве предложения 3.1 .

Обозначим ширину части 2 через $w, 1 \leqslant w \leqslant q$. Для удобства рассмотрения дополним эту часть бланка до квадрата $q \times q$ (см. рис. 5 ). Тогда в первой строке полученной таблицы будет находиться рассмотренная вьше перестановка $\pi$, а в остальных строках - все ее различные циклические перестановки (ср. доказательство леммы 4.1). Заштрихованные клетки на рис. 5 содержат элементы из $T$. Ясно, что для произвольного бланка перестановкой строк можно добиться того, чтобы эти элементы размещались “лесенкой”, как в примере, показанном на рис. 5. Ниже мы будем считать, что в рассматриваемой части вида 2 такая перестановка строк произведена.

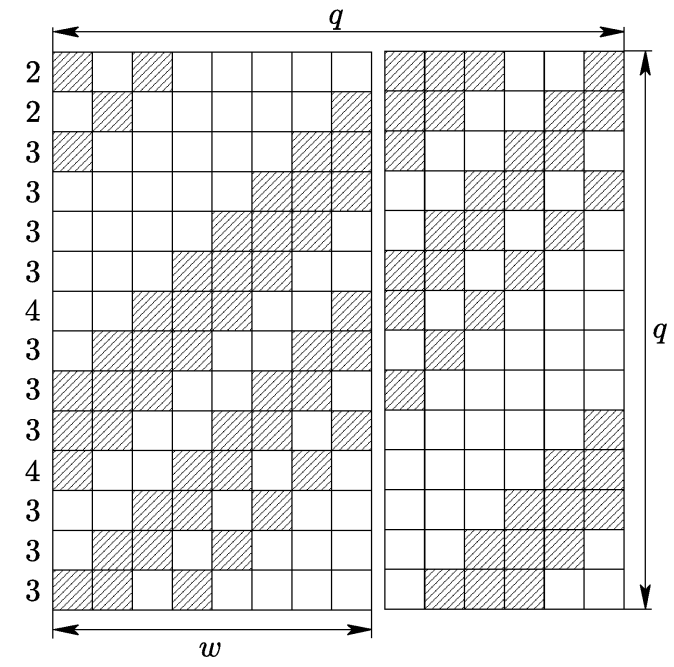

Рис. 5. Пример “дополненной” части 2 (номера $Q$-классов не проставлены). Элементы $T$ соответствуют заштрихованным клеткам, элементы $S$ - пустым. Слева указаны характеристические числа строк 
Множеству $T$ поставим в соответствие слово $Z_{T}$ длины $q$ над алфавитом $\{0,1\}$, которое получается из первой строки “дополненной" части 2 заменой элементов $T$ на 1 и элементов $S$ на 0 . Обозначим через $Z_{T}(w, j)$ префикс длины $w$ циклической перестановки слова $Z_{T}$, начинающейся с $j$-го символа. Слова $Z_{T}(w, j)$ соответствуют всем $q$ строкам рассматриваемой части 2. Характеристическим числом $j$-й строки назовем величину

$$
\chi(j)=\min \left\{\left|Z_{T}(w, j)\right|_{0},\left|Z_{T}(w, j)\right|_{1}\right\},
$$

где $\left|Z_{T}(w, j)\right|_{0},\left|Z_{T}(w, j)\right|_{1}$ - соответственно число нулей и единищ в слове $Z_{T}(w, j)$. Для $j$-й строки части $2 \chi(j)$ равно минимальному количеству джокеров, необходимому в этой строке для того, чтобы расстановка была допустимой. Характеристическим числом множества $T$ назовем величину

$$
\chi(T)=\sum_{j=1}^{q} \chi(j) .
$$

Таким образом, $\chi(T)$ равно минимальному числу джокеров в части 2 в допустимой расстановке при данном множестве $T$.

ЛЕмма 4.2. 1) Eсли $t \leqslant\lfloor w / 2\rfloor$, то $\chi(T)=t w$ для любого множсества $T$.

2) Eсли $t>\lfloor w / 2\rfloor$, то $\chi(T)=\left\lfloor w^{2} / 2\right\rfloor$ дя любого множества $T$ такого, что $Z_{T}$ - чиклическая перестановка слова $1 \ldots 10 \ldots 0$.

Множество $T$ такое, что $Z_{T}$ - циклическая перестановка слова $1 \ldots 10 \ldots 0$, обозначим через $\bar{T}$.

ДокАЗАТЕЛЬСТво. Первое утверждение леммы очевидно. В самом деле, при $t \leqslant\lfloor w / 2\rfloor$ в каждом слове $Z_{T}(w, j)$ единиц не более $\lfloor w / 2\rfloor$, т.е. не больше, чем нулей. Следовательно, $\chi(j)=\left|Z_{T}(w, j)\right|_{1}$ для всякого $j$. Каждая единица из $Z_{T}$ встречается в $w$ словах $Z_{T}(w, j)$, а значит, всего в этих словах содержится $t w$ единиц, откуда $\chi(T)=t w$.

Докажем второе утверждение. “Дополненная" часть 2 в случае множества $\bar{T}$ перестановкой строк приводится к виду, показанному на рис. 6 .

Строки, в которых содержатся элементы как из $S$, так и из $T$, т. е. такие строки, в которых надо ставить джокеры, пересекаются с выделенными на рис. 6 частями $\mathbf{a}, \mathbf{b}, \mathbf{c}, \mathbf{d}$. Для строк, пересекающих часть а или часть $\mathbf{d}, \chi(j)=\left|Z_{T}(w, j)\right|_{1}$, т. е. джокерами необходимо заменять элементы множества $\bar{T}$ (это клетки частей $\mathbf{a}$ и $\mathbf{d})$. Для строк, пересекаюших часть $\mathbf{b}$ и (или) часть $\mathbf{c}, \chi(j)=\left|Z_{T}(w, j)\right|_{0}$ и джокерами необходимо заменять элементы множества $S$ (это клетки частей $\mathbf{b}$ и $\mathbf{c}$ ). Таким образом, $\chi(\bar{T})$ - суммарное число клеток в частях $\mathbf{a}, \mathbf{b}, \mathbf{c}$ и $\mathbf{d}$. Первое слагаемое приводимой формулы соответствует части $\mathbf{a}$, второе $-\mathbf{b}$, третье - $\mathbf{c}$, четвертое - $\mathbf{d}$ :

$$
\chi(\bar{T})=\sum_{j=1}^{\left\lfloor\frac{w}{2}\right\rfloor} j+\sum_{j=1}^{\left\lfloor\frac{w-1}{2}\right\rfloor} j+\sum_{j=1}^{\left\lfloor\frac{w-1}{2}\right\rfloor} j+\sum_{j=1}^{\left\lfloor\frac{w}{2}\right\rfloor} j .
$$

Выгисляя значение суммы при нечетном $w$, получаем

$$
\chi(\bar{T})=4 \cdot \frac{\left(1+\frac{w-1}{2}\right)\left(\frac{w-1}{2}\right)}{2}=\frac{w^{2}-1}{2}=\left\lfloor\frac{w^{2}}{2}\right\rfloor,
$$




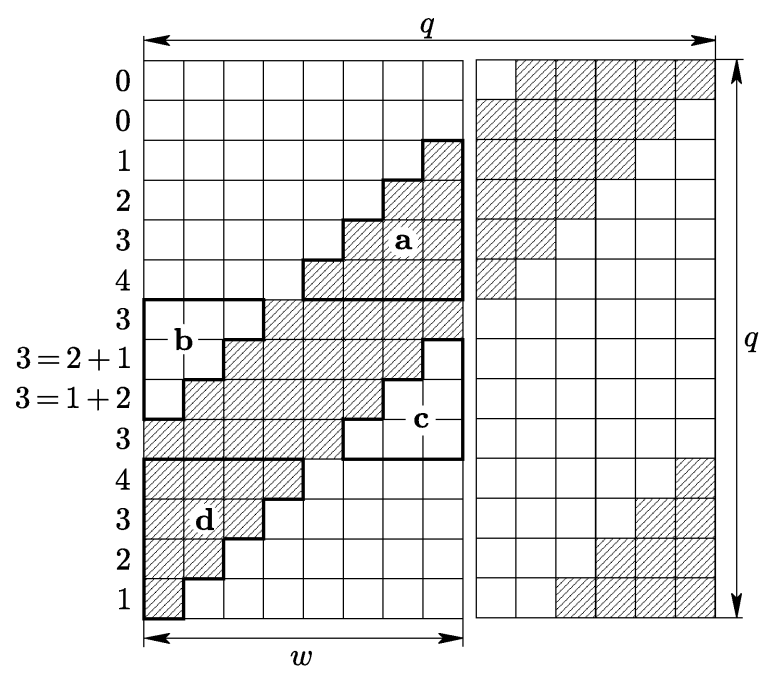

РИс. 6. Дополненная часть 2 для множества $\bar{T}$. Элементы $\bar{T}$ соответствуют заштрихованным клеткам. Слева указаны характеристические числа строк

а при четном $w$ имеем

$$
\chi(\bar{T})=2 \cdot \frac{\left(1+\frac{w-2}{2}\right)\left(\frac{w-2}{2}\right)}{2}+2 \cdot \frac{\left(1+\frac{w}{2}\right)\left(\frac{w}{2}\right)}{2}=\frac{w^{2}}{2}=\left\lfloor\frac{w^{2}}{2}\right\rfloor .
$$

ЛЕМмА 4.3. При любом фиксированном $t>\lfloor w / 2\rfloor$ выполнено равенство

$$
\min _{|T|=t} \chi(T)=\chi(\bar{T})
$$

где $\bar{T}$ таково, что $Z_{\bar{T}}-$ циклическая перестановка слова $1 \ldots 10 \ldots 0$.

ДокАЗАТЕльство. Вначале распространим определение слова $Z_{T}(w, j)$ на случай произвольного целого $j$, а именно определим $Z_{T}(w, j)$ как префикс длины $w$ циклической перестановки слова $Z_{T}$, начинающейся с позищии $j \bmod q$, а если $j \bmod q=0$, то с $q$-й позиции. Данное определение можно наглядно представить следующим образом: слово $Z_{T}$ - "циклическое", т. е. за его последней буквой снова следует первая и т. д. Тогда $j$-я позиция как раз и совпадает с позицией $j \bmod q$. Величину $\chi(j)$ при таком определении можно рассматривать как функцию $\mathbb{Z} \rightarrow \mathbb{N}$, периодическую с периодом $q$. Рассмотрим также вспомогательную функцию $\varphi(j)=\left|Z_{T}(w, j)\right|_{1}$ (это тоже периодическая с периодом $q$ функция $\left.\mathbb{Z} \rightarrow \mathbb{N}\right)$. Заметим, что для произвольного $m \in \mathbb{Z}$

$$
\begin{gathered}
\chi(j)=\left\{\begin{array}{l}
\varphi(j), \quad \varphi(j) \leqslant \frac{w}{2}, \\
w-\varphi(j), \quad \varphi(j)>\frac{w}{2},
\end{array}\right. \\
\sum_{j=m}^{m+q-1} \chi(j)=\chi(T), \quad \sum_{j=m}^{m+q-1} \varphi(j)=t w .
\end{gathered}
$$




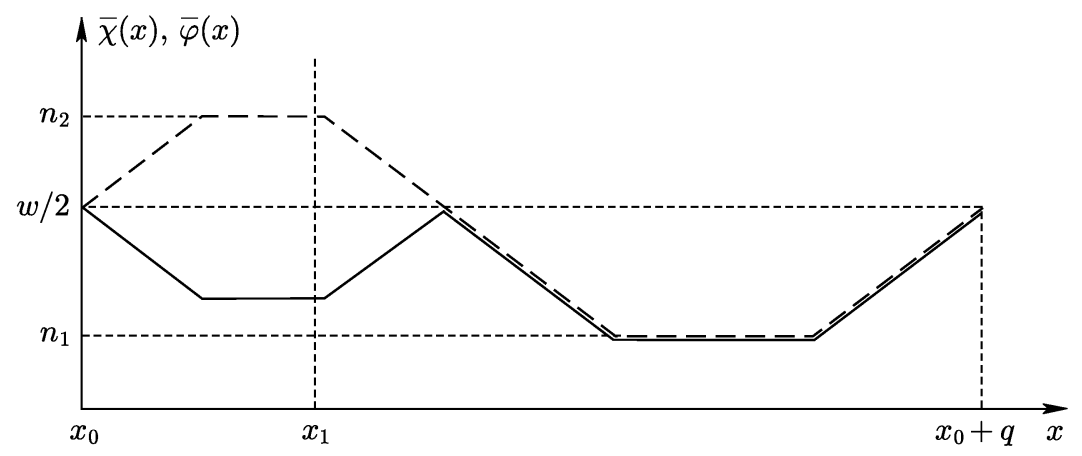

Рис. 7. Графики $\bar{\chi}(x)$ (сплошная линия) и $\bar{\varphi}(x)$ (штриховая линия) на периоде

Будет удобно доопределить функции $\varphi$ и $\chi$ на всей числовой прямой. $Ф$ ункцию $\varphi(x)$ определим как кусочно линейную, построенную по точкам $\varphi(j)$, а функцию $\chi(x)$ - заменив $j$ на $x$ в равенстве (4.1). Рассмотрим свойства и график функций $\varphi(x), \chi(x)$ (пример приведен на рис. 7 ).

1) Поскольку число единиц (как и число нулей) в соседних словах $Z_{T}(w, j)$ отличается не более чем на единицу, имеем для любого $j \in \mathbb{Z}$

$$
(\varphi(j+1)-\varphi(j)),(\chi(j+1)-\chi(j)) \in\{1,0,-1\},
$$

откуда следует, что любой линейньй участок графики $\varphi(x)$ или $\chi(x)$ имеет угловой коэффициент $-1,0$ или 1.

$2)$ Из (4.1) следует, что график $\chi(x)$ получается из графика $\varphi(x)$ отражением относительно прямой $y=w / 2$ той его части, которая вьше этой прямой.

3) Для любых $m \in \mathbb{Z}, x_{0} \in \mathbb{R}$ выполнено

$$
\begin{aligned}
& \int_{x_{0}}^{x_{0}+q} \varphi(x) d x=\int_{m}^{m+q} \varphi(x) d x=\sum_{j=m}^{m+q-1} \varphi(j)=t w, \\
& \int_{x_{0}}^{x_{0}+q} \chi(x) d x=\int_{m}^{m+q} \chi(x) d x=\sum_{j=m}^{m+q-1} \chi(j)=\chi(T) .
\end{aligned}
$$

Таким образом, при фиксированном $t$ константа $\int_{x_{0}}^{x_{0}+q} \varphi(x) d x$ не зависит от выбора множества $T$. Необходимо доказать, что константа $\int_{x_{0}}^{x_{0}+q} \chi(x) d x$, принимаюшая, вообще говоря, различные значения при различных $T$, минимальное значение принимает на множестве $\bar{T}$, указанном в условии леммы.

Рассмотрим функции $\bar{\varphi}(x), \bar{\chi}(x)$, соответствующие множеству $\bar{T}$. Тогда график функции $\bar{\varphi}(x)$ на некотором периоде будет иметь вид, приведенный на рис. 7 (штриховая линия). Константный промежуток со значением $\bar{\varphi}(x)=n_{2}$ соответствует максимальному количеству единиц в слове $Z_{\bar{T}}(w, j)$ (или максимальному количеству элементов $T$ в строке части 2 ), а константный промежуток со значением $\bar{\varphi}(x)=n_{1}$ соответствует минимальному количеству единиц в слове $Z_{\bar{T}}(w, j)$ (или минимальному количеству элементов $T$ в строке части 2 ). Согласно свойству 2) график $\bar{\chi}(x)$ будет иметь вид, изображенный на рис. 7 сплошной линией. 
Оценим константы $n_{1}$ и $n_{2}$. Пользуясь тем, что $Z_{\bar{T}}$ - циклическая перестановка слова $1 \ldots 10 \ldots 0$, выберем $j_{0}$ такое, что $Z_{\bar{T}}\left(w, j_{0}\right)$ является префиксом этого слова. Тогда $Z_{\bar{T}}\left(w, j_{0}\right)$ содержит максимальное количество (т. е. $\left.n_{2}\right)$ единиц. Если $w \leqslant t$, то слово $Z_{\bar{T}}\left(w, j_{0}\right)$ целиком состоит из единиц, откуда $n_{2}=w$. Если же $t<w$, то данное слово содержит все $t$ единиц, имеюшихся в $Z_{\bar{T}}$, откуда $n_{2}=t$. В итоге $n_{2}=\min \{w, t\}$. Рассуждая аналогично, получаем $n_{1}=\max \{0, w-s\}$. Заметим, что для любого множества $T(|T|=t)$ всякое слово $Z_{T}(w, j)$ содержит не более чем $t$ и не более чем $w$ единиц. Следовательно, $\varphi(x) \leqslant n_{2}$ для любого рассматриваемого $T$ и для любого $x \in \mathbb{R}$. Аналогично приходим к выводу, что $\varphi(x) \geqslant n_{1}$.

Кроме того, из условия $t \leqslant s$ следует, что $n_{1} \leqslant w-n_{2}$, т. е. левый (на рис. 7 ) локальньй минимум функции $\bar{\chi}(x)$ находится вьше правого. В самом деле, если $n_{2}=w$, то $t \geqslant w$, откуда $s \geqslant w$ и $n_{1}=0$. Если же $n_{2}=t<w$, то $w-t \geqslant w-s$ и $w-t>0$, откуда $w-t \geqslant n_{1}$.

Обозначим $\bar{X}_{\geqslant}=\{x \mid \bar{\varphi}(x) \geqslant w / 2\}, \bar{X}_{<}=\{x \mid \bar{\varphi}(x)<w / 2\}$. Тогда

$$
\int_{x_{0}}^{x_{0}+q} \bar{\chi}(x) d x=\int_{\bar{X}_{\geqslant}} \bar{\chi}(x) d x+\int_{\bar{X}_{<}} \bar{\chi}(x) d x=\int_{x_{0}}^{x_{1}} \bar{\chi}(x) d x+\int_{x_{1}}^{x_{0}+q} \bar{\chi}(x) d x .
$$

Теперь рассмотрим произвольное слово $Z_{T}$ с условием $|T|=t$, а также соответствуюшие ему функции $\varphi(x)$ и $\chi(x)$ и докажем, что

$$
\int_{x_{0}}^{x_{0}+q} \bar{\chi}(x) d x \leqslant \int_{x_{0}}^{x_{0}+q} \chi(x) d x .
$$

Обозначим $X_{\geqslant}=\{x \mid \varphi(x) \geqslant w / 2\}, X_{<}=\{x \mid \varphi(x)<w / 2\}$. Условимся также обозначать через $\rho(M)$ длину (или меру) множества $M$, где $M$ - объединение конечного числа непересекающихся промежутков из отрезка $\left[x_{0}, x_{0}+q\right]$.

Как показано вьше, $n_{1} \leqslant \varphi(x) \leqslant n_{2}$. Если $\varphi(x) \leqslant w / 2$ всегда, то

$$
\int_{x_{0}}^{x_{0}+q} \chi(x) d x=\int_{x_{0}}^{x_{0}+q} \varphi(x) d x=t w>\left\lfloor\frac{w^{2}}{2}\right\rfloor=\int_{x_{0}}^{x_{0}+q} \bar{\chi}(x) d x .
$$

Пусть существует точка, где $\varphi(x)>w / 2$. Если для любого $y$ выполнено неравенство

$$
\rho(\{x \mid \chi(x) \geqslant y\}) \geqslant \rho(\{x \mid \bar{\chi}(x) \geqslant y\})
$$

то тривиально

$$
\int_{x_{0}}^{x_{0}+q} \chi(x) d x \geqslant \int_{x_{0}}^{x_{0}+q} \bar{\chi}(x) d x
$$

Предположим, что $\int_{x_{0}}^{x_{0}+q} \chi(x) d x<\int_{x_{0}}^{x_{0}+q} \bar{\chi}(x) d x$. Тогда существует $y_{0}$ такое, что $\rho\left(\left\{x \mid \chi(x)<y_{0}\right\}\right)>\rho\left(\left\{x \mid \bar{\chi}(x)<y_{0}\right\}\right)$.

Оценим значение $y_{0}$. Из непрерывности функции $\varphi(x)$ следует, что она по крайней мере дважды на периоде принимает значение $w / 2$; в тех же точках $\chi(x)=w / 2$, т. е. $\chi(x)$ на периоде хотя бы дважды достигает максимального значения. Заметим, что функция $\bar{\chi}(x)$ достигает значения $w / 2$ ровно дважды на периоде (в точках $x_{0}$ и $\left.x_{1}\right)$ и опускается до локальных минимумов с наибольшей возможной скоростью - 
с постоянным угловым коэффициентом, равным -1. Следовательно, для любого $y \geqslant w-n_{2}$ (как было доказано, это больший локальньй минимум функции $\left.\bar{\chi}(x)\right)$ имеем

$$
\rho(\{x \mid \chi(x)<y\}) \leqslant \rho(\{x \mid \chi(x)<y\}) .
$$

Таким образом, $y_{0}<w-n_{2}$. Но в этом случае значения, не превосходящие $y_{0}$, функция $\chi(x)$ принимает только в области $X_{<}$, а функция $\bar{\chi}(x)-$ в области $\bar{X}<$. В силу свойств функции $\bar{\chi}(x)$ в области $\bar{X}_{<}=\left(x_{1} ; x_{0}+q\right)$ (см. рис. 7$)$ из неравенства

$$
\rho\left(\left\{x \mid \chi(x)<y_{0}\right\} \cap X_{<}\right)>\rho\left(\left\{x \mid \bar{\chi}(x)<y_{0}\right\} \cap \bar{X}_{<}\right)
$$

следует неравенство

$$
\rho\left(\{x \mid \chi(x)<y\} \cap X_{<}\right)>\rho\left(\{x \mid \bar{\chi}(x)<y\} \cap \bar{X}_{<}\right)
$$

для любого $y \leqslant w / 2$. Таким образом, $\rho\left(X_{<}\right)>\rho\left(\bar{X}_{<}\right)$. Но тогда $\rho\left(\bar{X}_{\geqslant}\right)>\rho\left(X_{\geqslant}\right)$, и из свойств функции $\varphi(x)$ в области $\bar{X}_{\geqslant}$(см. рис. 7 ) имеем

$$
\int_{\bar{X}_{\geqslant}}\left(\bar{\varphi}(x)-\frac{w}{2}\right) d x \geqslant \int_{X_{\geqslant}}\left(\varphi(x)-\frac{w}{2}\right) d x .
$$

Таким образом,

$$
\begin{aligned}
t w & =\int_{x_{0}}^{x_{0}+q} \varphi(x) d x=\int_{x_{0}}^{x_{0}+q} \chi(x) d x+2 \int_{X_{\geqslant}}\left(\varphi(x)-\frac{w}{2}\right) d x \\
& <\int_{x_{0}}^{x_{0}+q} \bar{\chi}(x) d x+2 \int_{\bar{X}_{\geqslant}}\left(\bar{\varphi}(x)-\frac{w}{2}\right) d x=\int_{x_{0}}^{x_{0}+q} \bar{\varphi}(x) d x=t w .
\end{aligned}
$$

Итак, предположение $\int_{x_{0}}^{x_{0}+q} \chi(x) d x<\int_{x_{0}}^{x_{0}+q} \bar{\chi}(x) d x$ привело нас к противоречию. Таким образом, лемма 4.3 доказана.

Из лемм 4.2, 4.3 вытекает, что

$$
k_{2}^{*}(t)= \begin{cases}t w, & t \leqslant\left\lfloor\frac{w}{2}\right\rfloor, \\ \left\lfloor\frac{w^{2}}{2}\right\rfloor, & t>\left\lfloor\frac{w}{2}\right\rfloor,\end{cases}
$$

т. е. график функции $k_{2}^{*}(t)$ выглядит так, как показано на рис. 8.

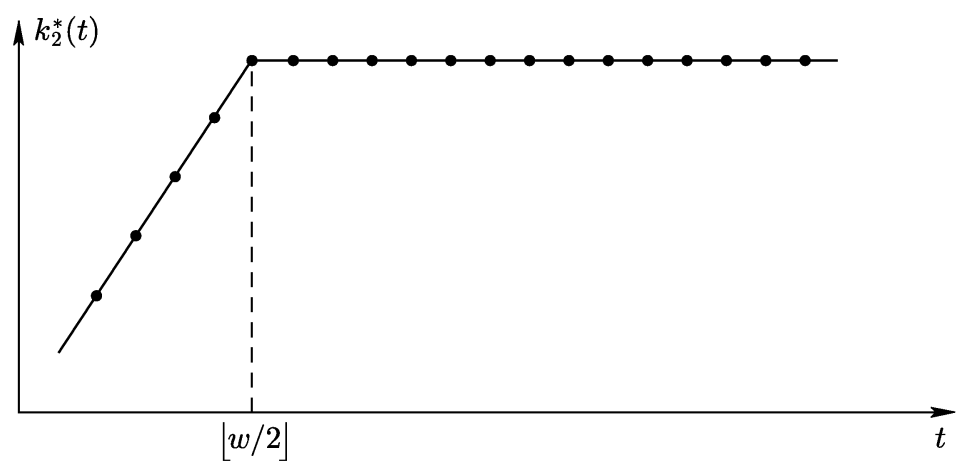

Рис. 8. График функции $k_{2}^{*}(t)$ 
ЛЕмма 4.4. Для любого $2 \leqslant t \leqslant\lfloor q / 2\rfloor$ справедливо:

a) $w \geqslant 4 \Longrightarrow k_{2}^{*}(t)-k_{2}^{*}(1) \geqslant w$;

б) $w=3 \Longrightarrow k_{2}^{*}(t)-k_{2}^{*}(1)=1$;

в) $w \leqslant 2 \Longrightarrow k_{2}^{*}(t)-k_{2}^{*}(1)=0$.

ДоказАтельство. а) $w \geqslant 4$. Поскольку $k_{2}^{*}(t)$ не убывает, то

$$
k_{2}^{*}(t)-k_{2}^{*}(1) \geqslant k_{2}^{*}(2)-k_{2}^{*}(1)=2 w-w=w .
$$

б) $w=3$. Тогда $\lceil w / 2\rceil=2$ и

$$
k_{2}^{*}(t)-k_{2}^{*}(1)=k_{2}^{*}(2)-k_{2}^{*}(1)=\left\lfloor\frac{3^{2}}{2}\right\rfloor-3=1 .
$$

в) $w=1$ или $w=2$. Тогда $\lceil w / 2\rceil=1$, откуда $k_{2}^{*}(2)=k_{2}^{*}(1)$.

Лемма 4.5. Для любого $2 \leqslant t \leqslant\lfloor q / 2\rfloor$ справедливо $k_{3}^{*}(1)-k_{3}^{*}(t) \leqslant 1$.

ДокАЗАТЕЛЬСТво. Рассмотрим часть 3 (см. рис. 4). При любом одноэлементном множестве $T$ в каждой строке находится не более одного элемента из $T$, а значит, в ней можно обойтись не более чем одним джокером. В некоторых строках оптимальной для части $\mathbf{3}$ расстановки при $t>1$ может не быть джокеров; мы укажем допустимую расстановку при $t=1$, в которой джокер стоит только в одной из таких строк. Тем самым лемма будет доказана.

Пусть значение $k_{3}^{*}(t), t>1$, достигается на множестве $T^{\prime}$. Если $j$-я строка не содержит джокеров при соответствующей расстановке, то $Z_{T^{\prime}}(w, j)=1 \ldots 1$ или $Z_{T^{\prime}}(w, j)=0 \ldots 0$. Выберем в $Z_{T^{\prime}}$ какую-нибудь единицу, входящую в подслово 01 или 10. Среди строк, в которые попадает $Q$-класс, соответствуюший этой единище, не более чем в одной нет джокеров. Для $t=1$ возьмем множество $T$, состоящее из выбранного $Q$-класса. Соответствуюшая ему расстановка является искомой.

ПримеР 4.1. Для бланка $(40,11,8) k_{3}^{*}(1)=1, k_{3}^{*}(3)=0$ (достигается при $T=\{0,2,5\} ;$ см. рис. $9, a)$. Более того, заметим, что при указанном $T$ минимальное количество джокеров в частях 3 и 4 в сумме равно нулю, в то время как при $t=1$ в частях 3 и 4 в совокупности нужно разместить два джокера.

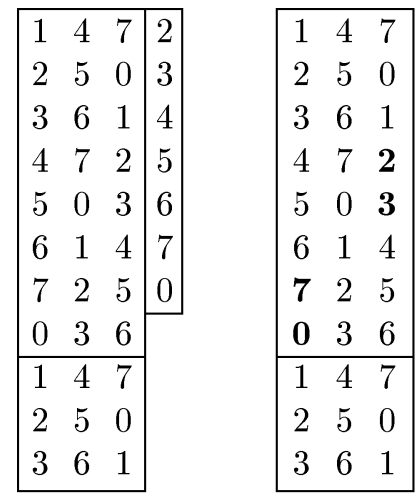

$a$ 6

Рис. 9. Бланки $(40,11,8)$ и $(33,11,8)$ 
ДОКАЗАТЕЛЬСТВО ПРЕДЛОЖЕНИЯ 3.1. ДЛя Любого фиксированного бланка $(L, p, q)$ и любого $t>1$ выполнено неравенство

$$
k^{*}(t) \geqslant\lfloor L /(p q)\rfloor k_{1}^{*}(t)+k_{2,1}^{*}(t)+\cdots+k_{2,\lfloor p / q\rfloor}^{*}(t)+k_{3}^{*}(t),
$$

где $k_{2,1}^{*}(t), \ldots, k_{2,\lfloor p / q\rfloor}^{*}(t)$ - количества джокеров в оптимальных при $t$ расстановках для соответствующих частей 2 . Обозначим сумму в правой части выписанного неравенства через $\sigma(t)$. Построим в бланке специальную расстановку (назовем ее оптимизированной), количество джокеров в которой не превосходит $\sigma(t)$. Тем самым, с учетом леммы 3.1 , предложение будет доказано.

Любую специальную расстановку в бланке можно построить по следующему алгоритму:

1) выбрать некоторый $Q$-класс в качестве единственного элемента $T$;

2) заменить джокерами все вхождения выбранного элемента в бланк;

3) выбрать в бланке некоторую строку;

4) в выбранной строке удалить все джокеры, поставленные ранее, и поставить джокеры во всех оставшихся позициях.

Заметим, что пересечение расстановки, полученной на шаге 2), с любой частью 2 бланка является расстановкой для этой части, оптимальной при $t=1$, а ее пересечение с частью 4 бланка содержит не более одного джокера. Пересечение итоговой специальной расстановки с любой частью 1 бланка также является расстановкой для этой части, оптимальной при $t=1$.

Оптимизированную расстановку построим следующим образом: $Q$-класс выберем так, чтобы минимизировать число джокеров в части $\mathbf{3}$, а строку - так, чтобы увеличение числа джокеров на шаге 4) в бланке было минимальным. Отметим, что выбор в каждом из случаев может быть не единственным, т. е. для данного бланка может существовать несколько оптимизированных расстановок; зафиксируем одну из них. Количество джокеров в построенной расстановке обозначим через $k$.

Количество джокеров в каждой части 1 оптимизированной расстановки равно $k_{1}^{*}(1)$. Количество джокеров в хвосте оптимизированной расстановки есть $k_{2,1}^{*}(1)+\cdots+k_{2,\lfloor p / q\rfloor}^{*}(1)+k_{3}^{*}(1)+k_{4}+\delta$, где $k_{4}-$ количество джокеров, поставленных на шаге 2 ) в части 4 , а $\delta$-минимальная разность между количеством элементов из $S$ и элементов из $T$ в одной строке в хвосте бланка.

Заметим, что специальная расстановка, не являющаяся оптимизированной, не может содержать менее $k$ джокеров: в ней может быть на джокер меньше только в части $\mathbf{4}$, но обязательно имеется дополнительньй джокер в части $\mathbf{3}$ и (или) дополнительный джокер за счет увеличения $\delta$.

Необходимо доказать, что

$$
\begin{aligned}
k- & \sigma(t)= \\
= & \underbrace{\left\lfloor\frac{L}{p q}\right\rfloor\left(k_{1}^{*}(1)-k_{1}^{*}(t)\right)+\left(k_{2,1}^{*}(1)-k_{2,1}^{*}(t)\right)+\cdots+\left(k_{2,\lfloor p / q\rfloor}^{*}(1)-k_{2,\lfloor p / q\rfloor}^{*}(t)\right)}_{\Delta_{1}} \\
& +\underbrace{\left(k_{3}^{*}(1)-k_{3}^{*}(t)\right)+k_{4}+\delta}_{\Delta_{2}} \leqslant 0 .
\end{aligned}
$$


Величина $\Delta_{1}$ является суммой неположительных слагаемых согласно леммам 4.1 и 4.4. Поэтому вначале оценим величину $\Delta_{2}$. Если в хвосте бланка нет элементов из $T$, то $k_{3}^{*}(1)=k_{4}=\delta=0$, т. е. $\Delta_{2} \leqslant 0$ и требуемое неравенство доказано. Предположим, что в хвосте есть элементы из $T$, а значит, на шаге 2 ) построения специальной расстановки в хвосте были поставлены джокеры. По построению $k_{4} \leqslant 1$, а по лемме $4.5\left(k_{3}^{*}(1)-k_{3}^{*}(t)\right) \leqslant 1$. Оценим $\delta$. В хвосте бланка есть "длинные" строки, состоящие из $\lceil(L \bmod p q) / p\rceil$ позиций, и "короткие" - из $\lfloor(L \bmod p q) / p\rfloor$ позиций. Если на шаге 2$)$ был поставлен джокер в "короткой" строке, то $\delta=\lfloor(L \bmod p q) / p\rfloor-2$ и, таким образом, $\Delta_{2} \leqslant\lfloor(L \bmod p q) / p\rfloor$. Если же на шаге 2) в "коротких" строках не появилось джокеров, то $\delta=\lfloor(L \bmod p q) / p\rfloor-1$; но в этом случае $k_{3}^{*}(1)=0$ (часть 3 состоит только из "коротких" строк). Следовательно, и в этом случае $\Delta_{2} \leqslant\lfloor(L \bmod p q) / p\rfloor$.

Теперь оценим $\Delta_{1}$, используя условие $L \geqslant 3 p+q$. Если в бланке есть часть $\mathbf{1}$, то по лемме 4.1

$$
\Delta_{1} \leqslant k_{1}^{*}(1)-k_{1}^{*}(t) \leqslant 2-p .
$$

Поскольку $\Delta_{2} \leqslant q-1$, получаем, что требуемое условие $\Delta_{1}+\Delta_{2} \leqslant 0$ выполнено.

Если же в бланке нет части $\mathbf{1}$, то в нем должна присутствовать часть 2 ширины не менее 4 . Тогда по лемме 4.4 имеем

$$
\Delta_{1} \leqslant k_{2,1}^{*}(1)-k_{2,1}^{*}(t) \leqslant-w,
$$

где $w=\lceil(L \bmod p q) / p\rceil$. Условие $\Delta_{1}+\Delta_{2} \leqslant 0$ снова выполнено. Предложение доказано.

ЗАмЕчАниЕ 4.1. Оценка для $L$, приводимая в условии предложения 3.1 , может быть незначительно понижена. Однако точная оценка сильно зависит от соотношения между конкретными $p$ и $q$. Отметим, что при $p>2 q$ условие $L \geqslant 2(p+q)$ является достаточным для существования специальной оптимальной расстановки (такая длина бланка обеспечивает наличие либо части $\mathbf{1}$, либо части $\mathbf{2}$ ширины не менее 4, либо двух частей 2 ширины 3 ; в последнем случае применение леммы 4.4 также позволяет доказать предложение 3.1$)$. В то же время в бланке $(33,11,8)$ (см. рис. 9,6$)$ любая специальная расстановка содержит не менее пяти джокеров, а оптимальная (при $T=\{0,2,5\}$ ) - четыре джокера, поставленные вместо номеров, выделенных жирным шрифтом, т.е. условие $L \geqslant 3 p$ в общем случае не является достаточным.

Из следуюшего предложения получим решение обратной задачи. Через $L^{\prime}$ обозначим длину хвоста бланка $(L, p, q)$, равную $L \bmod p q$.

ПРЕДЛОЖЕНИЕ 4.1. Для произвольного бланка $(L, p, q)$ такого, что $q=3$, $L \geqslant 2 p-2$ или $q \geqslant 4, L \geqslant 3 p+q$, выполнено:

a) ecлu $L^{\prime}<q$, mo $k^{*}=(p+q-2)\left\lfloor\frac{L}{p q}\right\rfloor$;

b) если $q \leqslant L^{\prime}<p$, mo $k^{*}=(p+q-2)\left\lfloor\frac{L}{p q}\right\rfloor+\left\lfloor\frac{L^{\prime}}{q}\right\rfloor-1$;

c) если $L^{\prime} \geqslant p, m o$

$$
(p+q-2)\left\lfloor\frac{L}{p q}\right\rfloor+\left\lfloor\frac{L^{\prime}}{q}\right\rfloor+\left\lfloor\frac{L^{\prime}}{p}\right\rfloor-2 \leqslant k^{*} \leqslant(p+q-2)\left\lfloor\frac{L}{p q}\right\rfloor+\left\lfloor\frac{L^{\prime}(p+q)}{p q}\right\rfloor-2 .
$$


ДоКАЗАТЕЛЬСТВО. Согласно предложению 3.1 и следствию 3.1 достаточно подсчитать джокеры в оптимальной специальной расстановке; сделаем это в предположении того, что такая расстановка построена по алгоритму, указанному в доказательстве предложения 3.1. Каждая часть 1 бланка содержит $p+q-2$ джокеров при любой специальной расстановке. Минимальное количество джокеров во всем бланке, таким образом, достигается при минимуме джокеров в его хвосте.

В случае а) выберем элемент множества $T$ так, чтобы он не встречался в хвосте бланка, а строку, в которой джокерами заменяются элементы из $S$, так, чтобы она не пересекала хвост бланка (и то, и другое возможно, поскольку хвост содержит менее $q$ символов). Тогда в хвосте не будет ни одного джокера и мы получим указанную в случае а) оценку.

Поскольку всякий номер $Q$-класса встречается в бланке один раз в каждых последовательных $q$ позициях, в случае b) выберем элемент множества $T$ так, что на шаге 2) построения расстановки в хвосте будет поставлено $\left\lfloor L^{\prime} / q\right\rfloor$ джокеров. Все они находятся в разных строках (каждая строка пересекается с хвостом бланка не более чем по одному символу). На шаге 3 ) выберем строку, содержащую джокер в хвосте, после чего на шаге 4) количество джокеров в хвосте уменьшится на 1. Отсюда следует требуемая оценка.

В случае с) заметим, что оценки снизу и сверху для $k^{*}$, в зависимости от параметров бланка, либо равны, либо различаются на 1. Первое слагаемое в обеих оценках есть количество джокеров в теле бланка, одинаковое для всех специальных расстановок. Аналогично случаю b) выберем элемент из $T$ так, что в хвосте бланка на шаге 2) ставится $\left\lfloor L^{\prime} / q\right\rfloor$ джокеров. Далее, на шаге 3) выберем строку, имеющую джокер в хвосте (тогда количество джокеров, добавляемых на шаге 4), будет меньше, чем если выбрать строку без джокеров в хвосте). При этом на шаге 4) в хвост бланка будет добавлено $\left\lfloor L^{\prime} / p\right\rfloor-2$ или $\left.\mid L^{\prime} / p\right\rfloor-1$ джокеров в зависимости от того, "короткая" или "длинная" строка выбрана. В итоге если найдется номер $Q$-класса, который встречается в хвосте бланка только $\left\lfloor L^{\prime} / q\right\rfloor$ раз и при этом хотя бы один раз - в "короткой" строке, то $k^{*}$ совпадает со значением левой части; если же всякий такой номер встречается только в “длинных" строках, то $k^{*}$ больше на единицу. Докажем, что в последнем случае

$$
\left\lfloor\frac{L^{\prime}(p+q)}{p q}\right\rfloor=\left\lfloor\frac{L^{\prime}}{q}\right\rfloor+\left\lfloor\frac{L^{\prime}}{p}\right\rfloor+1
$$

тем самым будет завершено доказательство случая с) и всего предложения.

Пусть $L^{\prime} \bmod q=r$. Тогда $q-r$ символов встречаются в хвосте "редко", т.е. $\left\lfloor L^{\prime} / q\right\rfloor$ раз. Поскольку эти символы встречаются только в “длинных" строках, то количество "коротких" строк не превосходит $r$ (первые символы нижних $r$ строк в хвосте бланка, очевидно, различны). С другой стороны, $L^{\prime} \bmod p$ равно количеству “длинных" строк, т. е. $p-r$. (Случай $r=0$ невозможен, так как при этом число $L^{\prime}$, меньшее $p q$ по определению, оказывается общим кратным взаимно простых $p$ и q.) В итоге получаем

$$
\begin{aligned}
\left\lfloor\frac{L^{\prime}(p+q)}{p q}\right\rfloor & =\left\lfloor\frac{L^{\prime}}{q}+\frac{L^{\prime}}{p}\right\rfloor=\left\lfloor\frac{L^{\prime}}{q}\right\rfloor+\left\lfloor\frac{L^{\prime}}{p}\right\rfloor+\left\lfloor\frac{L^{\prime} \bmod q}{q}+\frac{L^{\prime} \bmod p}{p}\right\rfloor \\
& =\left\lfloor\frac{L^{\prime}}{q}\right\rfloor+\left\lfloor\frac{L^{\prime}}{p}\right\rfloor+\left\lfloor\frac{r}{q}+\frac{p-r}{p}\right\rfloor=\left\lfloor\frac{L^{\prime}}{q}\right\rfloor+\left\lfloor\frac{L^{\prime}}{p}\right\rfloor+1 .
\end{aligned}
$$




\section{§5. Доказательство теоремы 2.1}

Прежде всего, отметим, что функция $k^{*}=k^{*}(L, p, q)$, которую мы умеем вычислять (предложение 4.1$)$, и длина взаимодействия $L(k, p, q)$ связаны следуюшим соотношением:

$$
L(k, p, q)=\min \left\{L \mid k^{*}(L, p, q)=k+1\right\} .
$$

Поскольку утверждение предложения 4.1 справедливо при некоторых ограничениях на $L$, необходимо проверить, что эти ограничения выполнены при имеющихся в теореме условиях, налагаемых на $k$. Для удобства изложения эта проверка будет проведена в конще доказательства.

Докажем утверждение п. b) теоремы. Покажем, что

$$
k^{*}(L+p q, p, q)=k^{*}(L, p, q)+(p+q-2) .
$$

В самом деле, бланки $(L+p q, p, q)$ и $(L, p, q)$ имеют одинаковые хвосты, а значит, в них при оптимальной специальной расстановке размешается одно и то же количество джокеров. Тело первого из бланков содержит на один блок больше - в этом блоке будет размешено $p+q-2$ джокеров, что и требовалось. Отсюда следует, что

$$
\min \left\{L \mid k^{*}(L, p, q)=k+1\right\}=\min \left\{L \mid k^{*}(L+p q, p, q)=k+p+q-1\right\} .
$$

Из соотношения (5.1) получаем

$$
L(k+p+q-2, p, q)=L(k, p, q)+p q,
$$

откуда

$$
\begin{aligned}
\Delta( & +p+q-2, p, q)=L(k+p+q-2, p, q)-\frac{p q}{p+q-2}(k+p+q-2) \\
\quad & L(k, p, q)+p q-\frac{p q}{p+q-2} k-p q=L(k, p, q)-\frac{p q}{p+q-2} k=\Delta(k, p, q) .
\end{aligned}
$$

Далее через $L^{\prime}$ обозначим длину хвоста бланка, а через $k^{\prime}$ - количество джокеров в хвосте, т. е. предположим, что для некоторого $\beta$ выполнено

$$
\begin{aligned}
L & =\beta p q+L^{\prime}, & & L^{\prime}<p q, \\
k & =\beta(p+q-2)+k^{\prime}, & & k^{\prime}<p+q-2 .
\end{aligned}
$$

Докажем утверждение основного пункта теоремы - п. а). Вначале докажем более простое неравенство.

Получим оценку снизу. С учетом (5.1) достаточно показать, что в хвосте бланка длины $L^{\prime}=\left\lfloor\frac{p q\left(k^{\prime}+1\right)}{p+q-2}-1\right\rfloor$ оптимальная расстановка содержит не более $k^{\prime}$ джокеров. Согласно п. 3) предложения 4.1 эта расстановка содержит в хвосте не более $\left\lfloor\frac{L^{\prime}(p+q)}{p q}\right\rfloor-2$ джокеров. Осталось заметить, что

$$
\left\lfloor\frac{L^{\prime}(p+q)}{p q}\right\rfloor-2=k^{\prime}+1+\left\lfloor\frac{2 k^{\prime}+1}{p+q-2}\right\rfloor-2 \leqslant k^{\prime} .
$$

Оценка снизу доказана. 
Получим оценку сверху. Вначале докажем, что данное неравенство справедливо в трех частных случаях, после чего покажем, что при любом значении $k$ примени́м один из этих частных случаев. Параметры $w, \alpha$ и $\gamma$ являются натуральными числами.

1. Пусть $L^{\prime}=\alpha q$, а $w \geqslant 2$ таково, что $w p=\alpha q+\gamma, \gamma<p$. Тогда имеем $k^{\prime} \geqslant \alpha+(w-1)-2=\alpha+w-3$ по предложению 4.1. Из соотношения (5.1) следует, что для любого $\beta$

$$
L(\beta(p+q-2)+\alpha+w-4, p, q) \leqslant \beta p q+\alpha q .
$$

Тогда

$$
\begin{aligned}
\Delta & (\beta(p+q-2)+\alpha+w-4, p, q) \\
& \leqslant \beta p q+\alpha q-\frac{p q}{p+q-2}(\beta(p+q-2)+\alpha+w-4) \\
& =\alpha q-\frac{p q}{p+q-2}(\alpha+w-4)=\alpha q-\frac{(q \alpha+\gamma) q}{q \alpha+(\gamma+(q-2) w)}(\alpha+w-4) \\
& =\alpha q-q \frac{q \alpha^{2}+(q w-4 q+\gamma) \alpha+(w-4) \gamma}{q \alpha+(\gamma+(q-2) w)}=q \frac{(4 q-2 w) \alpha-(w-4) \gamma}{q \alpha+(\gamma+(q-2) w)} \\
& =4 q-2 w-\frac{(4 q-2 w)((q-2) w+\gamma)+(w-4) q \gamma}{q \alpha+(\gamma+(q-2) w)} .
\end{aligned}
$$

Знаменатель последней дроби положителен; докажем, что положителен и числитель. Числитель является квадратным трехчленом относительно $q$, после раскрытия скобок приводящимся к виду

$$
4 w q^{2}+\left(\gamma w-8 w-2 w^{2}\right) q+\left(4 w^{2}-2 \gamma w\right)
$$

Его дискриминант после упрощений оказьвается равен

$$
D=w^{2}(2 w-(\gamma+8))^{2},
$$

что позволяет вычислить корни $q_{1}=(2 w-\gamma) / 4$ и $q_{2}=2$. Поскольку $q_{1}<q$ по определению $w$ и $\gamma, q_{2}<q$ по условию теоремы и коэффициент при $q^{2}$ положителен, то квадратный трехчлен при допустимых значениях $q$ положителен, а значит, положительна и вся дробь. Таким образом,

$$
\Delta(\beta(p+q-2)+\alpha+w-4, p, q)<4 q-2 w \leqslant 4(q-1) .
$$

2. Пусть $L^{\prime}=w p, w \geqslant 2$, а $\alpha$ таково, что $w p=\alpha q+\gamma, \gamma<q$. Тогда $k^{\prime} \geqslant \alpha+$ $w-2$ по предложению 4.1. Воспользовавшись (5.1), получаем для любого $\beta$

$$
L(\beta(p+q-2)+\alpha+w-3, p, q) \leqslant \beta p q+w p
$$


и производим аналогичные выкладки для $\Delta$ :

$$
\begin{aligned}
& \Delta(\beta(p+q-2)+\alpha+w-3, p, q) \\
& \quad \leqslant \beta p q+w p-\frac{p q}{p+q-2}(\beta(p+q-2)+\alpha+w-3) \\
& \quad=\alpha q+\gamma-\frac{p q}{p+q-2}(\alpha+w-3)=\alpha q+\gamma-\frac{(q \alpha+\gamma) q}{q \alpha+(\gamma+(q-2) w)}(\alpha+w-3) \\
& \quad=\alpha q+\gamma-q \frac{q \alpha^{2}+(q w-3 q+\gamma) \alpha+(w-3) \gamma}{q \alpha+(\gamma+(q-2) w)}=\gamma+q \frac{(3 q-2 w) \alpha-(w-3) \gamma}{q \alpha+(\gamma+(q-2) w)} \\
& \quad=\gamma+3 q-2 w-\frac{(3 q-2 w)((q-2) w+\gamma)+(w-3) q \gamma}{q \alpha+(\gamma+(q-2) w)} .
\end{aligned}
$$

Снова знаменатель последней дроби положителен, а числитель является квадратным трехчленом относительно $q$; после раскрытия скобок он приводится к виду

$$
3 w q^{2}+\left(\gamma w-6 w-2 w^{2}\right) q+\left(4 w^{2}-2 \gamma w\right) .
$$

Вьчисляя дискриминант, получаем

$$
D=w^{2}(2 w-(\gamma+6))^{2}
$$

отсюда находим корни $q_{1}=(2 w-\gamma) / 3$ и $q_{2}=2$. Как и в случае 1, при допустимых значениях $q$ квадратный трехчлен положителен, а значит, положительна и вся дробь. Таким образом,

$$
\Delta(\beta(p+q-2)+\alpha+w-3, p, q)<\gamma+3 q-2 w \leqslant 4(q-1) .
$$

3. Пусть $L^{\prime}=\alpha q$ и $p=\alpha q+\gamma$ (т.е. $\left.w=1\right)$. В этом случае в хвост бланка необходимо поставить $\alpha-1$ джокеров согласно п. 2) предложения 4.1. Снова пользуясь (5.1), для любого $\beta$ имеем

$$
L(\beta(p+q-2)+\alpha-2, p, q) \leqslant \beta p q+\alpha q
$$

и проводим вычисления для $\Delta$ :

$$
\begin{aligned}
& \Delta(\beta(p+q-2)+\alpha-2, p, q) \leqslant \beta p q+\alpha q-\frac{p q}{p+q-2}(\beta(p+q-2)+\alpha-2) \\
& \quad=\alpha q-\frac{p q}{p+q-2}(\alpha-2)=\alpha q-\frac{(q \alpha+\gamma) q}{q \alpha+(q+\gamma-2)}(\alpha-2) \\
& \quad=\alpha q-q \frac{q \alpha^{2}+(\gamma-2 q) \alpha-2 \gamma}{q \alpha+(q+\gamma-2)}=q \frac{(3 q-2) \alpha+2 \gamma}{q \alpha+(q+\gamma-2)} \\
& \quad=3 q-2-\frac{(3 q-2)(q+\gamma-2)-2 q \gamma}{q \alpha+(q+\gamma-2)} .
\end{aligned}
$$

Знаменатель последней дроби положителен, числитель приводится к виду $3 q^{2}+$ $(\gamma-8) q-2 \gamma+4$ с корнями $q_{1}=(2-\gamma) / 3, q_{2}=2$. Снова при всех допустимых значениях $q$ дробь положительна, откуда

$$
\Delta(\beta(p+q-2)+\alpha-2, p, q)<3 q-2<4(q-1) .
$$


Теперь возьмем произвольное $k=\beta(p+q-2)+k^{\prime}, k^{\prime}<p+q-2$. Докажем, что $\Delta(k, p, q)<4(q-1)$. Если $k^{\prime}+2<p / q$, положим $\alpha=k^{\prime}+2, \gamma=p-\alpha q$ и применим случай 3 , получая неравенство (5.4).

Пусть $k^{\prime}+2>p / q$. Предположим, что найдется такое $\alpha$, что $k^{\prime}+4=\alpha+\left\lceil\frac{\alpha q}{p}\right\rceil$. Тогда, положив $w=\left\lceil\frac{\alpha q}{p}\right\rceil$ и $\gamma=w p-\alpha q$, можно применить случай 1 и вывести неравенство (5.2).

Предположим теперь, что указанное выше значение $\alpha$ не сушествует. Поскольку значения выражения $\alpha+\left\lceil\frac{\alpha q}{p}\right\rceil$ с ростом $\alpha$ на единицу увеличивается не более чем на 2 , то сушествует такое $\alpha$, что

$$
\begin{gathered}
\alpha+\left\lceil\frac{\alpha q}{p}\right\rceil=k^{\prime}+3 \\
(\alpha+1)+\left\lceil\frac{(\alpha+1) q}{p}\right\rceil=k^{\prime}+5 .
\end{gathered}
$$

Положим $w=\left\lceil\frac{\alpha q}{p}\right\rceil$ и $\gamma=w p-\alpha q$ и убедимся в применимости случая 2. В самом деле, если $w=\left\lceil\frac{\alpha q}{p}\right\rceil=1$, получаем $k^{\prime}+2=\alpha<p / q$. Противоречие. Отсюда имеем $w \geqslant 2$. Из соотношений (5.5) и (5.6) следует, что $\left\lceil\frac{(\alpha+1) q}{p}\right\rceil>\left\lceil\frac{\alpha q}{p}\right\rceil$, откуда $\gamma<q$. Итак, случай 2 применим. Получаем неравенство (5.3). Доказательство п. а) завершено.

c) Произведем выкладки, аналогичные приведенным в п. а). Пусть $p=\alpha q+1$ и $L^{\prime}=2 \alpha q$. Тогда всякий $Q$-класс встречается в хвосте ровно $2 \alpha$ раз, т. е. на шаге 2 ) построения специальной расстановки в хвосте будет поставлено $2 \alpha$ джокеров. В качестве $T$ можно выбрать класс, встречающийся в строке длины 1 , после чего на шаге 3) выбрать эту строку. Тогда на шаге 4) количество джокеров уменьшится на 1. В итоге получим $k^{\prime}=2 \alpha-1$. Если же $L^{\prime}=2 \alpha q-1$, то выберем $T=\{0\}$ (этот класс встречается в хвосте $2 \alpha-1$ раз, в том числе в одноэлементной строке, а именно в предпоследней, в силу выбора $p$ ). Таким образом, можно ограничиться $2 \alpha-2$ джокерами. Из (5.1) следует, что для любого $\beta$

$$
L(\beta(p+q-2)+(2 \alpha-2), p, q)=\beta p q+2 \alpha q .
$$

Тогда

$$
\begin{aligned}
& \Delta(\beta(p+q-2)+2 \alpha-2, p, q)=\beta p q+2 \alpha q-\frac{p q}{p+q-2}(\beta(p+q-2)+2 \alpha-2) \\
& \quad=2 \alpha q-\frac{p q}{p+q-2}(2 \alpha-2)=2 \alpha q-\frac{(q \alpha+1) q}{q \alpha+1+q-2}(2 \alpha-2) \\
& \quad=2 \alpha q-2 q \frac{q \alpha^{2}-(q-1) \alpha-1}{q \alpha+(q-1)}=2 q \frac{2(q-1) \alpha+1}{q \alpha+(q-1)}=4(q-1)-\frac{4(q-1)^{2}-2 q}{q \alpha+(q-1)} .
\end{aligned}
$$

Последняя дробь положительна, а при увеличении $\alpha$ она стремится к нулю. Таким образом, при любом заданном $\varepsilon>0$ и заданном $q$ можно указанным способом выбрать $p$ и $k$ так, чтобы

$$
4(q-1)-\varepsilon<\Delta(k, p, q)<4(q-1) .
$$


d) Из предложения 4.1 имеем $k^{*}(\beta p q-1, p, q)=\beta(p+q-2)-1$ и $k^{*}(\beta p q, p, q)=$ $\beta(p+q-2)$. Из соотношения (5.1) получаем, что если $k=\beta(p+q-2)-1$, то $L(k, p, q)=\beta p q$. Тогда $\Delta(k, p, q)=p q /(p+q-2)$ и с учетом оценки снизу из п. а), утверждение d) доказано.

е) Пусть $k=\beta(p+q-2)$. Из предложения 4.1 получаем $L(k, p, q)=\beta p q+2 q$ и $\Delta(k, p, q)=\beta p q+2 q-\beta p q=2 q$.

Мы доказали все пункты теоремы. Осталось доказать правомерность применения предложения 4.1, т. е. выполнение приведенных в нем ограничений на $L$ при заданных в теореме ограничениях на $k$. Пусть вначале $q=3$. Тогда

$$
k^{*}(2 p-2, p, 3) \leqslant\left\lfloor\frac{(2 p-2)(p+3)}{3 p}\right\rfloor-2=\left\lfloor\frac{2 p+1}{3}-\frac{2}{p}\right\rfloor-1=\left\lfloor\frac{2 p}{3}\right\rfloor-1 \text {. }
$$

Это означает, что

$$
L\left(\left\lfloor\frac{2 p}{3}\right\rfloor-1, p, q\right) \geqslant 2 p-2,
$$

что и требовалось. Если же $q \geqslant 4$, то

$$
k^{*}(3 p+q, p, q) \leqslant\left\lfloor\frac{(3 p+q)(p+q)}{p q}\right\rfloor-2 \leqslant\left\lfloor\frac{3 p}{q}\right\rfloor+3 .
$$

Отсюда получаем требуемое условие

$$
L\left(\left\lfloor\frac{3 p}{q}\right\rfloor+3, p, q\right) \geqslant 3 p+q .
$$

Доказательство теоремы завершено.

Авторы благодарны Е. В. Суханову и В. Ю. Попову за многочисленные ценные замечания.

\section{Список литературы}

1. Berstel J., Boasson L. Partial words and a theorem of Fine and Wilf // Theor. Comp. Sci. 1999. V. 218. P. 135-141.

2. Fine N.J., Wilf H.S. Uniqueness theorem for periodic functions // Proc. Amer. Math. Soc. 1965. V. 16. P. 109-114.

3. Choffrut C., Karhumäki J. Combinatorics on words // Handbook of formal languages. V. 1. Ch.6. Berlin: Springer, 1997. P. 329-438.

4. Shur A. M., Konovalova Yu. V. On the periods of partial words // Lect. Notes Comp. Sci. 2001. V. 2136. P. 657-665.

5. Blanchet-Sadri F., Hegstrom R. A. Partial words and a theorem of Fine and Wilf revisited // Theor. Comp. Sci. 2002. V. 270. P. 401-419.

6. Colosimo A., De Luca A. Special factors in biological strings // J. Theor. Biol. 2000. V. 204. P. 29-46.

7. Head T., Păun G., Pixton D. Language Theory and Molecular Genetics // Handbook of formal languages. V. 2. Ch.7. Berlin: Springer, 1997. P. 295-360. 